\title{
Noise Propagation in Multiple-Input ADC-Based Measurement Systems
}

\author{
Diego Bellan \\ Department of Electronics, Information and Bioengineering, Politecnico di Milano, \\ piazza Leonardo da Vinci, 32, 20133, Milan, Italy, diego.bellan@polimi.it
}

\begin{abstract}
In this paper, the complete statistical characterization of the amplitude spectrum at the output of a multiple-input ADC-based measurement system is derived under the assumption of input channels with different noise levels. In practical applications the input channels correspond to the spatial components of a vector field (e.g., magnetic/electric field). Each output spectral line represents the amplitude of the vector field at a specific frequency. Such amplitude is a random variable depending on the noise levels (internal and external noise) of the input channels. Closed form analytical solution for the probability density function of the vector field amplitude is not available in the mathematical literature under the hypothesis of different noise levels. Therefore, an analytical expression for the probability density function is derived on the basis of a Laguerre series expansion. The impact of the kind of time window, the sampling frequency, and the number of samples is clearly derived and put into evidence. Approximate analytical expressions for the mean value and the variance of the vector field amplitude are also provided. Analytical results are validated by means of numerical simulations.
\end{abstract}

Keywords: Statistics, noise, analog-to-digital conversion, discrete Fourier transform, frequency domain analysis.

\section{INTRODUCTION}

$\mathrm{M}$ EASUREMENT SYSTEMS based on analog-to-digital conversion of signals are widely used in several engineering fields. The increasing availability of faster and higher-resolution analog-to-digital converters (ADCs), together with the increasing computational capability of processors, allow the design and development of measurement systems with increasing complexity. An interesting example of such complexity is represented by an ADC-based measurement system designed to measure the frequency content of a non-stationary vector field (e.g., magnetic/electric field) [1]-[3]. In fact, in this case the measurement system must implement one input channel for each spatial component of the vector field (e.g., the $x, y$, and $z$ components) to be acquired simultaneously. Then, the digitized signals must be transformed into the frequency domain and properly processed to provide the required information concerning the whole vector field. Typically, if the vector field to be measured is non-stationary in time, each measurement window consists of a small number of samples. In this case it is well known that the noise effects, coming from both internal (i.e., electronic) and external (i.e., environmental) sources, can greatly affect the measurement accuracy [4]. The analysis of noise propagation from the analog amplitude domain to the frequency domain is therefore a fundamental task in order to provide a proper statistical characterization of the whole measurement process.

Noise propagation in single-channel ADC-based measurement systems has been widely studied in the relevant technical literature (e.g., [5]-[7]). For the multipleinput case, however, the available literature is mainly concerning the system architecture (e.g., [8]), whereas a thorough statistical analysis of noise propagation has not been provided yet. The main difficulty is related to the circumstance that, in the most general case, the input channels are characterized by different noise levels (i.e., internal and/or external noise). In fact, the whole vectorfield measurement in the frequency domain requires a proper processing of input data affected by different noise levels. A complete statistical characterization of the measurement results would require the derivation of the probability density function (PDF) of the measured field amplitude. Such an objective, however, is still a challenging issue from a mathematical viewpoint. Indeed, in the case of different noise levels, the amplitude of the measured vector field is a random variable (RV) whose PDF has not been yet derived in analytical closed form in the mathematical literature (e.g., [9]-[10]). In this paper, an analytical approach based on a Laguerre series expansion [10] will be exploited for deriving the PDF of the vector field amplitude measured by an ADC-based multiple-input measurement system with different noise levels per channel [11]. The impact of specific signal processing choices, such as the kind of time window, the number of samples, and the sampling frequency, will be put into evidence.

The paper is organized as follows. In Section 2 the motivations of the paper are reported, with the description of an ADC-based multiple-input measurement system, and the required data processing to obtain the vector field amplitude in the frequency domain. In Section 3 the mathematical results concerning the Laguerre series expansion are reported and used for a complete statistical description of a measured vector-field amplitude. Moreover, approximate results concerning the mean value and the variance of the vector field amplitude are reported, while the related derivations are provided in the Appendix. In Section 4 the analytical results are compared with numerical results obtained by means of properly designed repeated-run simulations. Finally, concluding remarks are drawn in Section 5 . 


\section{MOTIVATION OF THE WORK}

The general analysis proposed in this work was motivated by the measurement application outlined in this Section.

Let us consider a measurement system for a three-axial vector field (e.g., a magnetic field) based on analog-todigital conversion of time-domain data. The measurement system is aimed at providing the amplitude spectrum of the vector field through a time-to-frequency transformation of the acquired data.

The three-dimensional nature of the field requires a threeaxial probe providing the time-domain analog signals corresponding to the vector field components (i.e., $x, y$, and $z$ components). More specifically, each analog signal is the sum of the related field component, i.e., $v_{x}(t), v_{y}(t)$ or $v_{z}(t)$, and additive noise. It is assumed that each field component can be modeled as a sum of sine waves, while additive noise consists of internal (i.e., electronic) and external (i.e., environmental) noise. In the following sections, mathematical derivations related to the $x$ channel will be shown. Similar expressions hold for $y$ and $z$ channels.

The $x$-component of the vector field can be written as the sum of a constant term $A_{x, 0}$, and $N$ sine waves with amplitude $A_{x, n}$, frequency $f_{n}$, and phase $\vartheta_{n}$, i.e.,:

$$
v_{x}(t)=A_{x, 0}+\sum_{n=1}^{N} A_{x, n} \cos \left(2 \pi f_{n} t+\vartheta_{n}\right)
$$

Analog signals are captured via a suitable three-axial probe and acquired in the form of three digitized time-series $x[k], y[k]$, and $z[k]$ by means of analog-to-digital conversion. The digitized signal $x[k]$ coming from the $x$ channel can be written as:

$$
x[k]=x\left(k T_{S}\right)=v_{x}\left(k T_{S}\right)+n_{x}\left(k T_{S}\right), k=0, \ldots, N_{S}-1,
$$

where $T_{S}$ is the sampling interval and $N_{S}$ is the length of each time-series. Independent additive zero-mean noise $n_{x}$ introduced by the $x$-channel has been assumed in (2). The sampling frequency $f_{S}=1 / T_{S}$ must be chosen in order to fulfill the sampling theorem, i.e., $f_{S}>2 f_{N}$, where $f_{N}$ is the maximum frequency in the input signal spectra.

Proper time-windowing through a window function $w[k]$ is adopted in order to reduce spectral leakage [12], and a properly weighted Discrete Fourier Transform (DFT) is used -via the Fast Fourier Transform (FFT) algorithm- in order to obtain spectral estimates of sine wave components:

$$
X[n]=\frac{2}{N_{S} \mathrm{NPSG}} \operatorname{DFT}\{x[k] w[k]\},
$$

where NPSG is the Normalized Peak Signal Gain characterizing the selected time window.
It can be shown that, regardless of the distribution of additive noise, the real and the imaginary parts (i.e., $X_{R}$ and $\left.X_{I}\right)$ of the random variable (RV) $X[n]$ are normally distributed uncorrelated RVs [5], with mean values given by their deterministic components (i.e., unbiased RVs), and equal variances $\sigma_{X}^{2}=\sigma_{X_{R}}^{2}=\sigma_{X_{I}}^{2}$ depending on the power of the corrupting noise $n_{x}$. By assuming locally-white noise (i.e., flat noise in a proper frequency interval including the sine wave to be estimated) one obtains [13]:

$$
\sigma_{X}^{2}=\operatorname{ENBW} S_{n_{x}} \Delta f
$$

where ENBW is the Equivalent Noise Bandwidth of the selected time window, $S_{n_{x}}$ is the local value of the noise Power Spectral Density (PSD), and $\Delta f=f_{S} / N_{S}$ is the DFT frequency resolution. Notice that, for a given PSD noise level, the frequency-domain variance $\sigma_{X}^{2}$ is affected by the kind of time window (i.e., the ENBW), and by the ratio between the sampling frequency and the number of samples (i.e., $\Delta f$ ).

In order to estimate the amplitude of the sinusoidal components of the measured field, the contributions from all the three channels must be taken into account. Thus, for each frequency index $n$, the measured sine-wave amplitude is given by:

$$
M[n]=\sqrt{X_{R}[n]^{2}+X_{I}[n]^{2}+Y_{R}[n]^{2}+Y_{I}[n]^{2}+Z_{R}[n]^{2}+Z_{I}[n]^{2}}
$$

where the subscripts $R$ and $I$ denote the real and the imaginary part of the related $R V$, respectively.

In the most general case considered in this paper, the noise levels of the three channels can take different values, i.e., $\sigma_{X}^{2} \neq \sigma_{Y}^{2} \neq \sigma_{Z}^{2}$. The reason can come from measurement channels with non-equal internal (electronic) noise levels, and/or from external (environmental) noise with different levels on the three axial components.

The circumstance of different noise levels results in a nontrivial problem when the statistical characterization of (5) is needed (e.g., PDF). In fact, while the case of equal variances is a standard statistical problem, the case of different variances is still unsolved in a closed analytical form. In the following Section, the statistics of $M$ will be derived under the general assumption of channels with different noise levels, by exploiting one of the last contributions in the mathematical literature resorting to a proper series expansion [10].

\section{MATHEMATICAL DERIVATION}

For the purpose of deriving the statistics of the RV (5) it is useful to derive first the PDF of the RV under the square root, i.e.,

$$
W=X_{R}^{2}+X_{I}^{2}+Y_{R}^{2}+Y_{I}^{2}+Z_{R}^{2}+Z_{I}^{2} .
$$


As a second step, the PDF and the CDF (i.e., the cumulative distribution function) of $M$ are obtained by means of the theorem on the transformation of RVs [14]. In fact, by observing that

$$
M=\sqrt{W},
$$

it follows that the PDF and the CDF are given by

$$
\begin{gathered}
f_{M}(M)=2 M f_{W}\left(M^{2}\right) \\
F_{M}(M)=F_{W}\left(M^{2}\right) .
\end{gathered}
$$

In the literature, several attempts have been made to derive the PDF of the sum of the squares of normal RVs with different variances and non-zero mean values. Different approximate expressions have been therefore derived, but the closed form analytical solution is not available yet. One of the more recent and interesting contributions in the mathematical literature is described in [9]. In that paper, the following $\mathrm{RV}$ is considered:

$$
W=\sum_{i=1}^{K} \alpha_{i}\left(z_{i}+\delta_{i}\right)^{2}
$$

where $z_{i}(i=1, \ldots, K)$ are standard normal RVs (i.e., zeromean and unit variance normal RVs). Notice that the coefficients $\delta_{i}$ and $\alpha_{i}$ take into account the actual mean values and variances of the $K$ RVs. In particular, by comparing (10) with (6) it can be easily observed that for the specific application under analysis we have $K=6$, and the coefficients are given by:

$$
\begin{gathered}
\alpha_{1}=\alpha_{2}=\sigma_{X}^{2} \\
\alpha_{3}=\alpha_{4}=\sigma_{Y}^{2} \\
\alpha_{5}=\alpha_{6}=\sigma_{Z}^{2} \\
\delta_{1}=X_{0 R} / \sigma_{X}, \quad \delta_{2}=X_{0 I} / \sigma_{X} \\
\delta_{3}=Y_{0 R} / \sigma_{Y}, \quad \delta_{4}=Y_{0 I} / \sigma_{Y} \\
\delta_{5}=Z_{0 R} / \sigma_{Z}, \quad \delta_{6}=Z_{0 I} / \sigma_{Z}
\end{gathered}
$$

where the subscript " 0 " denotes the mean value of the corresponding RV.

In [10] the PDF of $W$ defined in (10) is expressed in the form of a Laguerre series expansion:

$$
f_{W}(\boldsymbol{\alpha} ; \boldsymbol{\delta} ; W)=\sum_{k=0}^{\infty} a_{k} \frac{k ! \Gamma(K / 2)}{\beta \Gamma(K / 2+k)} g\left(K ; \frac{W}{\beta}\right) L_{k}^{(K / 2-1)}\left(\frac{W}{2 \beta}\right),
$$

where $\boldsymbol{\alpha}=\left\{\alpha_{i}, i=1, \ldots, K\right\}$ and $\boldsymbol{\delta}=\left\{\delta_{i}, i=1, \ldots, K\right\}$ are the vectors of the coefficients defined above, the coefficients $a_{k}$ are calculated recursively as:

$$
\begin{aligned}
& a_{0}=1 \\
& a_{k}=\frac{1}{k} \sum_{r=0}^{k-1} b_{k-r} a_{r}, \quad k \geq 1
\end{aligned}
$$

and the coefficients $b_{k}$ are given by:

$$
b_{k}=-\frac{k}{2} \sum_{i=1}^{K} \delta_{i}^{2} \gamma_{i}^{k-1}+\frac{1}{2} \sum_{i=1}^{K}\left(1+k \delta_{i}^{2}\right) \gamma_{i}^{k}, \quad k \geq 1
$$

where

$$
\gamma_{i}=1-\alpha_{i} / \beta, \quad i=1, \ldots, K
$$

Expansion (12) is uniformly convergent for all $W>0$ provided that $\beta>\max _{k} \alpha_{k} / 2$.

Finally, in (12), the function $g(K ; x)$ is the central $\chi^{2}$ PDF with $K$ degrees of freedom given by:

$$
g(K ; x)=\frac{1}{2^{K / 2} \Gamma(K / 2)} x^{K / 2-1} \exp (-x / 2), \quad x \geq 0
$$

and $L_{k}^{(K / 2-1)}(x)$ is the $k$-th generalized Laguerre polynomial given by:

$$
L_{k}^{(n)}(x)=\sum_{m=0}^{k}\left(\begin{array}{c}
k+n \\
k-m
\end{array}\right) \frac{(-x)^{m}}{m !}
$$

with parameter $n=K / 2-1$.

The analytical expression for the PDF of the measured amplitude $M$ can be therefore obtained by means of (8) and (12) as:

$$
f_{M}(\boldsymbol{\alpha} ; \boldsymbol{\delta} ; M)=2 M \sum_{k=0}^{\infty} a_{k} \frac{k ! \Gamma(3)}{\beta \Gamma(3+k)} g\left(6 ; \frac{M^{2}}{\beta}\right) L_{k}^{(2)}\left(\frac{M^{2}}{2 \beta}\right)
$$

A series expansion for the CDF of $W$ is also derived in [10]:

$$
F_{W}(\boldsymbol{\alpha} ; \boldsymbol{\delta} ; W)=G\left(K ; \frac{W}{\beta}\right)+\sum_{k=1}^{\infty} a_{k} \frac{(k-1) !}{\Gamma(K / 2+k)}\left(\frac{W}{2 \beta}\right)^{K / 2} e^{-\frac{W}{2 \beta}} L_{k-1}^{(K / 2)}\left(\frac{W}{2 \beta}\right),(19
$$

where the function $G(K ; x)$ is the central $\chi^{2}$ CDF with $K$ degrees of freedom given by:

$$
G(K ; x)=1-e^{-x / 2} \sum_{k=0}^{K / 2-1} \frac{1}{k !}\left(\frac{x}{2}\right)^{k} .
$$

From (9) and (19), by letting $K=6$, the analytical expression for the CDF of the measured amplitude $M$ can be written as: 


$$
F_{M}(\boldsymbol{\alpha} ; \boldsymbol{\delta} ; M)=G\left(6 ; \frac{M^{2}}{\beta}\right)+\sum_{k=1}^{\infty} a_{k} \frac{(k-1) !}{\Gamma(3+k)}\left(\frac{M^{2}}{2 \beta}\right)^{3} e^{-\frac{W}{2 \beta}} L_{k-1}^{(3)}\left(\frac{M^{2}}{2 \beta}\right)
$$

The analytical results given by (18) and (21) provide a complete statistical characterization of the measured amplitude $M$. The statistical moments (e.g., the mean value and the variance) of the RV $M$ can be derived from (18).

However, approximate and simpler analytical results could be useful from the application viewpoint [15]. Approximate expressions for the mean value and the variance of $M$ can be readily obtained from the results derived in Appendix starting from a Taylor series approach [14]. From equations (A2), (A3) and (A4), for the mean value of $M$ it can be obtained:

$$
\mu_{M} \cong \sqrt{A^{2}+2\left(\sigma_{X}^{2}+\sigma_{Y}^{2}+\sigma_{Z}^{2}\right)}-\frac{\sigma_{X}^{2}\left(A_{X}^{2}+\sigma_{X}^{2}\right)+\sigma_{Y}^{2}\left(A_{Y}^{2}+\sigma_{Y}^{2}\right)+\sigma_{Z}^{2}\left(A_{Z}^{2}+\sigma_{Z}^{2}\right)}{2\left(A^{2}+2\left(\sigma_{X}^{2}+\sigma_{Y}^{2}+\sigma_{Z}^{2}\right)\right)^{3 / 2}},
$$

where:

$$
\begin{aligned}
& A_{X}^{2}=X_{0 R}^{2}+X_{0 I}^{2} \\
& A_{Y}^{2}=Y_{0 R}^{2}+Y_{0 I}^{2} \\
& A_{Z}^{2}=Z_{0 R}^{2}+Z_{0 I}^{2} \\
& A^{2}=A_{X}^{2}+A_{Y}^{2}+A_{Z}^{2}
\end{aligned}
$$

From (A3), (A4) and (A5), for the variance of $M$ it can be obtained:

$$
\sigma_{M}^{2} \cong \frac{\sigma_{X}^{2}\left(A_{X}^{2}+\sigma_{X}^{2}\right)+\sigma_{Y}^{2}\left(A_{Y}^{2}+\sigma_{Y}^{2}\right)+\sigma_{Z}^{2}\left(A_{Z}^{2}+\sigma_{Z}^{2}\right)}{A^{2}+2\left(\sigma_{X}^{2}+\sigma_{Y}^{2}+\sigma_{Z}^{2}\right)}
$$

Notice that (22) and (24) give also the mean value and the variance of only-noise spectral components provided that the amplitudes $A, A_{\mathrm{X}}, A_{\mathrm{Y}}$, and $A_{\mathrm{Z}}$ are set to zero.

\section{NUMERICAL ASSESSMENT}

Analytical results derived in Section 3 have been validated through properly designed numerical simulations. A threeaxial sinusoidal vector field with normalized amplitude $A=1$ has been simulated, consisting of three sinusoidal components with the same amplitude $A / \sqrt{3}$ and frequency $f=24.41 \mathrm{MHz}$. The three sine waves were corrupted by additive zero-mean white Gaussian noise with different PSD, i.e., $\quad S_{n_{x}}=70 \cdot 10^{-9}, \quad S_{n_{y}}=60 \cdot 10^{-9}, \quad$ and $S_{n_{z}}=50 \cdot 10^{-9}$. Notice that a noise distribution different from the Gaussian distribution could be considered since the normal behavior of the DFT coefficients (3) is due to the Central Limit Theorem [14] and not to the kind of noise distribution. Each noisy sine wave was sampled with sampling frequency $f_{S}=1 \mathrm{GS} / \mathrm{s}$, by taking a number of samples per channel $N_{S}=2^{12}$. Each digitized signal was then multiplied by a Hann window, and the DFT was evaluated by means of the FFT. According to (3) applied to each channel, the three frequency spectra were obtained. Notice that from (4) the frequency-domain variances resulting from the Hann window (i.e., $E N B W=1.5$ ) and the above mentioned noise data and sampling conditions are $\sigma_{X}^{2}=2.6 \cdot 10^{7}, \sigma_{Y}^{2}=2.2 \cdot 10^{7}$, and $\sigma_{Z}^{2}=1.8 \cdot 10^{7}$. Finally, according to (5), the frequency spectrum of the vector field was obtained. In Fig.1. an example of the noisy field spectrum in the frequency range $(0,100 \mathrm{MHz})$ is shown.

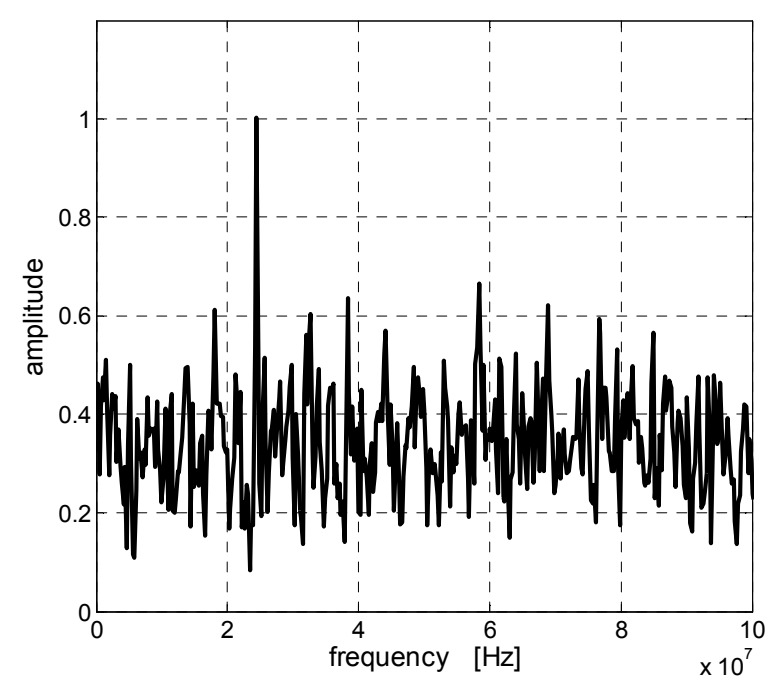

Fig.1. Example of amplitude spectrum of the simulated vector field consisting of a unit amplitude sine wave at $24.41 \mathrm{MHz}$ and additive noise with different levels on the three channels.

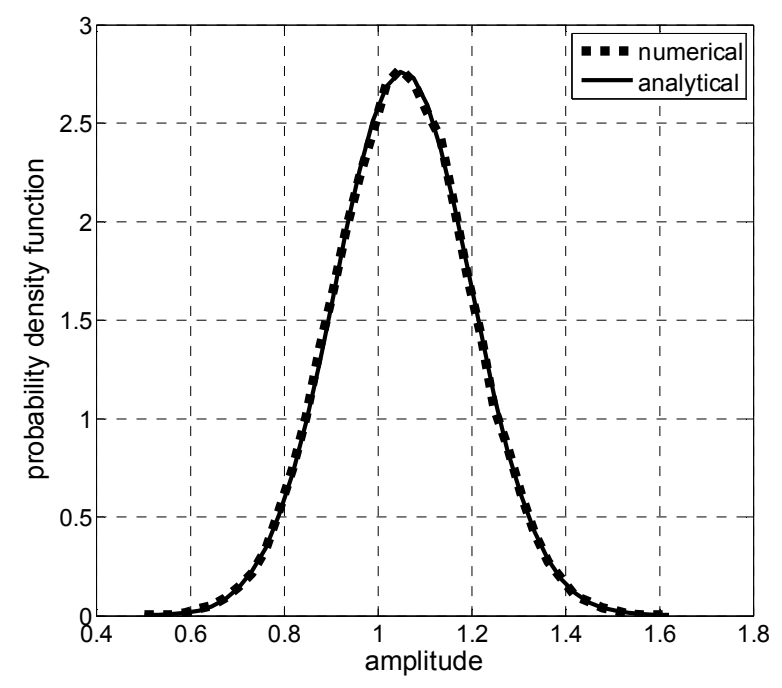

Fig.2. Comparison between the analytical PDF given by (18) and the numerical PDF obtained by repeated-run numerical simulations of the spectral line amplitude corresponding to the field sine wave at $24.41 \mathrm{MHz}$.

The analytical results obtained in Section 3 refer to each spectral line in the field spectrum. Therefore, by focusing on the spectral line corresponding to the field sine wave (i.e., the spectral line at $24.41 \mathrm{MHz}$ ), a repeated run analysis can be performed in order to obtain a numerical estimate of its statistical behavior. Fig.2. shows the numerical PDF of the measured variable $M$ (i.e., the measured sine wave amplitude according to (5)), obtained by $4 \cdot 10^{4}$ repeated simulation runs of the numerical procedure outlined above. 
On the same graph, the analytical PDF given by (18) is shown. It can be observed that, due to the noise bias, the mean value of the measured variable $M$ (i.e., the location of the peak of the symmetric bell-shaped curve) is larger than the noise-free value $A=1$.

In Fig. 3. the impact of the use of different time windows is shown. In particular, from (4) it is clear that a change in the selected time window results in a different value for the parameter ENBW, leading to different values for the frequency-domain variances. In Fig.3., three windows are compared, i.e., the rectangular window $(E N B W=1)$, the Hann window $(E N B W=1.5)$, and the minimum 4-term Blackman-Harris window ( $E N B W=2)$. As it was expected, the spread and the bias of the measured $M$ increase with the ENBW.

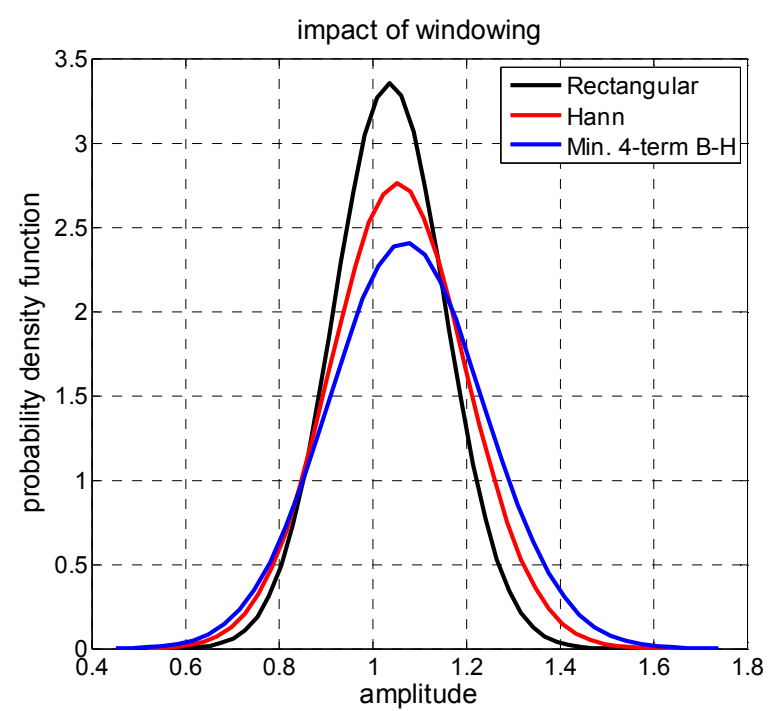

Fig.3. PDF of the field amplitude $M$ at $24.41 \mathrm{MHz}$ for different time windows. The spread and the bias increase with the $E N B W$ of the selected window.

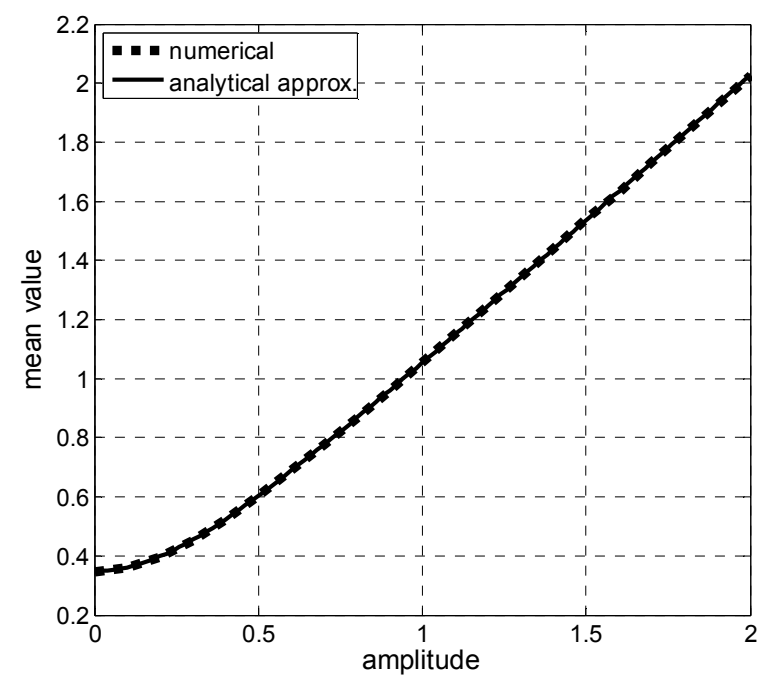

Fig.4. Analytical vs numerical behavior of the mean value of the simulated noisy field at $24.41 \mathrm{MHz}$. Noise levels and sampling conditions are the same as in Fig.2.
Fig.4. provides a comparison between the analytical approximate result given in (22) for the mean value of the spectral line amplitude at $24.41 \mathrm{MHz}$ and numerical estimates obtained through repeated-run simulations under the same conditions as in Fig.2. The mean values are evaluated for field amplitude $A$ ranging from 0 to 2 . Notice that as the field amplitude approaches zero, the mean value remains greater than zero due to the noise bias.

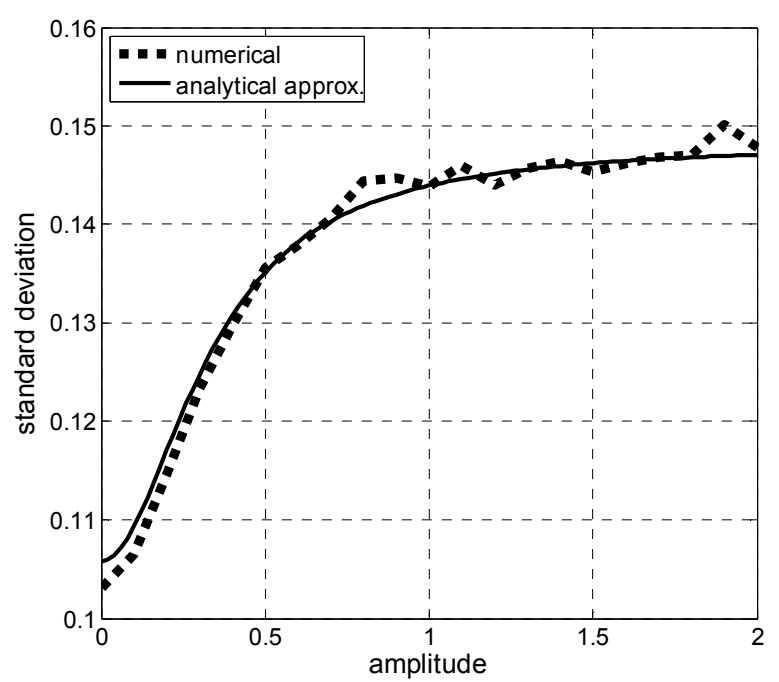

Fig.5. Analytical vs numerical behavior of the standard deviation of the simulated noisy field at $24.41 \mathrm{MHz}$. Noise levels and sampling conditions are the same as in Fig.2.

In Fig.5. the comparison is related to the standard deviation of the amplitude of the simulated field at $24.41 \mathrm{MHz}$, for the same noise levels and sampling conditions as in Fig.4. The analytical approximate standard deviation, given by the square root of (24), is compared to numerical results obtained by repeated-run simulations. The field amplitude ranges from 0 to 2 . As the amplitude approaches zero, the graph provides the standard deviation of a spectral line consisting of only noise. Notice also the change in the slope of the curve, i.e., a large slope for low amplitude field and a small slope for higher amplitudes.

\section{CONCLUSION}

In this paper, one of the more interesting and recent results available in the mathematical literature has been applied to the complete statistical characterization of the measured amplitude of a vector field by means of an ADC-based multiple-input measurement system with different noise levels per channel. The relationship with the sampling and windowing parameters has been shown and clarified. Although the analytical results have been applied to a threecomponent vector field in the work, the proposed approach has a general validity with respect to the number of input channels. 


\section{APPENDIX}

Let us consider the random variable

$$
z=f\left(y_{1}, \ldots, y_{6}\right)=\sqrt{\sum_{k=1}^{6} y_{k}}
$$

where $y_{1}, y_{2}, \ldots, y_{6}$ denote six uncorrelated random variables. By means of the theorem on the transformation of RVs [14], the mean value of $z$ can be estimated as follows:

$$
\begin{aligned}
\mu_{z} & \cong f\left(\mu_{y_{1}}, \ldots, \mu_{y_{6}}\right)+\left.\frac{1}{2} \sum_{k=1}^{6} \sigma_{y_{k}}^{2} \frac{\partial^{2} f}{\partial y_{k}^{2}}\right|_{\mu_{y_{1}}, \ldots, \mu_{y_{6}}} \\
& =\sqrt{\sum_{k=1}^{6} \mu_{y_{k}}}-\frac{\sum_{k=1}^{6} \sigma_{y_{k}}^{2}}{8\left(\sum_{k=1}^{6} \mu_{y_{k}}\right)^{\frac{3}{2}}}
\end{aligned}
$$

In the case where $y_{k}$ is the square of normally distributed RVs, i.e., $y_{k}=x_{k}^{2}$, it can be easily shown that [14]:

$$
\begin{gathered}
\mu_{y_{k}}=\mu_{x_{k}}^{2}+\sigma_{x_{k}}^{2}, \\
\sigma_{y_{k}}^{2}=2 \sigma_{x_{k}}^{2}\left(\sigma_{x_{k}}^{2}+2 \mu_{x_{k}}^{2}\right),
\end{gathered}
$$

By substitution of mean value (A3) and variance (A4) into (A2) the explicit expression of $\mu_{z}$ as a function of $\mu_{x_{k}}$ and $\sigma_{x_{k}}^{2}$ can be obtained.

The variance of $z$ can be estimated as follows [14]:

$$
\sigma_{z}^{2} \cong \sum_{k=1}^{6} \sigma_{y_{k}}^{2}\left(\frac{\partial f}{\partial y_{k}}\right)_{\mu_{y_{1}}, \ldots, \mu_{y_{6}}}^{2}=\frac{\sum_{k=1}^{6} \sigma_{y_{k}}^{2}}{4 \sum_{k=1}^{6} \mu_{y_{k}}}
$$

\section{REFERENCES}

[1] Bellan, D., Gaggelli, A., Maradei, F., Mariscotti, A., Pignari, S.A. (2004). Time-domain measurement and spectral analysis of nonstationary low-frequency magnetic-field emissions on board of rolling stock. IEEE Transactions on Electromagnetic Compatibility, 46 (1), 12-23.

[2] Krug, F., Mueller, D., Russer, P. (2004). Signal processing strategies with the TDEMI measurement system. IEEE Transactions on Instrumentation and Measurement, 53 (5), 1402-1408.
[3] Bellan, D., Gaggelli, A., Pignari, S.A. (2009). Noise effects in time-domain systems involving three-axial field probes for the measurement of nonstationary radiated emissions. IEEE Transactions on Electromagnetic Compatibility, 51 (2), 192-203.

[4] Kay, S.M. (1988). Modern Spectral Estimation: Theory \& Applications. Prentice-Hall.

[5] Schoukens, J., Renneboog, J. (1986). Modeling the noise influence on the Fourier coefficients after a discrete Fourier transform. IEEE Transactions on Instrumentation and Measurement, 35 (3), 278-286.

[6] Jenq, Y. (1988). Measuring harmonic distortion and noise floor of an A/D converter using spectral averaging. IEEE Transactions on Instrumentation and Measurement, 37 (4), 525-528.

[7] Solomon, O.M. (1992). The effects of windowing and quantization error on the amplitude of frequencydomain functions. IEEE Transactions on Instrumentation and Measurement, 41 (6), 932-937.

[8] Kim, Y., Tewfik, A.H., Gowreesunker, B.V. (2012). Multi-channel analog-to-digital conversion using a single-channel quantizer. In 20th European Signal Processing Conference (EUSIPCO). IEEE, 10441048.

[9] Kotz, S., Johnson, N.L., Boyd, D.W. (1967). Series representations of distributions of quadratic forms in normal variables II. Non-central case. Annals of Mathematical Statistics, 38, 838-848.

[10] Castano-Martinez, A., Lopez-Blazquez, F. (2005). Distribution of a sum of weighted noncentral chisquare variables. Sociedad de Estadistica $e$ Investigacion Operativa, 14 (2), 397-415.

[11] Bellan, D. (2010). Uncertainty effects of unequal noise levels in three-dimensional fields measurement. In International Conference on Signals and Electronic Systems (ICSES). IEEE, 443-446.

[12] Harris, F.J. (1978). On the use of windows for harmonic analysis with the discrete Fourier transform. Proceedings of the IEEE, 66 (1), 51-83.

[13] Bellan, D. (2013). Detection and estimation of weak sine waves with random offset and additive noise. In 3rd International Conference on Instrumentation, Communication, Information Technology, and Biomedical Engineering (ICICI-BME). IEEE, 156161.

[14] Papoulis, A., Pillai, S.U. (2002). Probability, Random Variables and Stochastic Processes ( $4^{\text {th }}$ ed.). McGrawHill.

[15] Bellan, D., Pignari, S.A. (2008). Noise influence in time-to-frequency transformation of radiated emissions data. In 8th International Symposium on Electromagnetic Compatibility - EMC Europe. IEEE, 731-734.

Received April 14, 2014. Accepted October 24, 2014. 